\title{
Existence Results for a Class of Fractional Differential Equations with Periodic Boundary Value Conditions and with Delay
}

\author{
Hadi Karami, ${ }^{1}$ Azizollah Babakhani, ${ }^{1}$ and Dumitru Baleanu ${ }^{2,3}$ \\ ${ }^{1}$ Department of Mathematics, Faculty of Basic Science, Babol University of Technology, Babol 47148-71167, Iran \\ ${ }^{2}$ Department of Mathematics and Computer Science, Cankaya University, Turkey \\ ${ }^{3}$ Institute of Space Sciences, P.O. Box, MG-23, 76900 Magurele-Bucharest, Romania
}

Correspondence should be addressed to Azizollah Babakhani; babakhani@nit.ac.ir

Received 11 June 2013; Accepted 15 August 2013

Academic Editor: Ali H. Bhrawy

Copyright (C) 2013 Hadi Karami et al. This is an open access article distributed under the Creative Commons Attribution License, which permits unrestricted use, distribution, and reproduction in any medium, provided the original work is properly cited.

We discuss the existence and uniqueness of solution for two types of fractional order ordinary and delay differential equations. Fixed point theorems are the main tool used here to establish the existence and uniqueness results. First we use Banach contraction principle to prove the uniqueness of solution and then Krasnoselskii's fixed point theorem to show the existence of the solution under certain conditions in a Banach space.

\section{Introduction}

In mathematics delay differential equations are a type of differential equation in which the derivative of unknown function at a certain time is given in terms of the values of the function at previous times.

While physical events such as acceleration and deceleration take little time compared to the times needed to travel most distances, times involved in biological processes such as gestation and maturation can be substantial when compared to the data-collection times in most population studies. Therefore, it is often imperative to explicitly incorporate these process times into mathematical models of population dynamics. These process times are often called delay times, and the models that incorporate such delay times are referred as delay differential equation models $[1,2]$.

Recently theory of fractional differential equations attracted many scientists and mathematicians to work on them [3-12]. For the existence of solutions for fractional differential equations, one can see [13-30] and references therein. The results have been obtained by using fixed point theorems like Picard's, Schauder fixed-point theorem, and Banach contraction mapping principle. About the development of existence theorems for fractional functional differential equations, many publications exist [31-35]. Many applications of fractional calculus amount to replacing the time derivative in a given evolution equation by a derivative of fractional order. The results of several studies clearly stated that the fractional derivatives seem to arise generally and universally from important mathematical reasons. Recently, interesting attempts have been made to give physical meaning to the initial conditions for fractional differential equations with Riemann-Liouville fractional derivatives which could be found in $[8,9,36,37]$.

Recently Benchoohra et al. [28] studied existence of solutions for a class of fractional differential equations with infinite delay; namely,

$$
\begin{gathered}
D^{\alpha} u(t)=u(t)+f\left(t, u_{t}\right), \quad \text { for each } t \in[0, b], 0<\alpha<1, \\
u(t)=\phi(t), \quad t \in[-\infty, 0],
\end{gathered}
$$

where $D^{\alpha}$ is the standard Riemann-Liouville fractional derivative and $f$ satisfies some assumptions.

First, in this paper we consider nonlinear delayed fractional differential equations:

$$
\begin{gathered}
D^{\alpha} u(t)=u(t)+f\left(t, u_{t}\right), \quad t \in(0,1), 0<\alpha<1, \\
u(t)=\phi(t), \quad t \in[-\tau, 0],
\end{gathered}
$$


associated with boundary conditions

$$
u(0)=\lim _{t \rightarrow 0^{+}} t^{1-\alpha} u(t)=u(1)
$$

where $D^{\alpha}$ is the standard Riemann-Liouville fractional derivative and $f$ is a continuous function. Here $u_{t}(\cdot)$ represents the properitoneal state from time $-\tau$ up to time $t$ which is defined by $u_{t}(\theta)=u(t+\theta),-\tau \leq \theta \leq 0$. We proved the uniqueness of existence solutions for (2) with periodic boundary condition (3) under some further conditions.

For investigating to establish an existence theorem, we also consider a class of nonlinear delayed fractional differential equations of the form

$$
\begin{gathered}
D^{\alpha} u(t)=u(t)+f(t, u(t), u(t-\tau)), \quad t \in(0,1), \\
u(t)=\phi(t), \quad t \in[-\tau, 0], 0<\alpha<1
\end{gathered}
$$

with periodic boundary condition

$$
\begin{gathered}
u(0)=\lim _{t \rightarrow 0^{+}} t^{1-\alpha} u(t)=c=u(1), \\
\left.D^{-(1-\alpha)} u(t)\right|_{t=0}=c \Gamma(\alpha) .
\end{gathered}
$$

The paper has been organized as follows. In Section 2 we give basic definitions and preliminary. Unique solution of (2)-(3) under some conditions is proved in Section 3. The existence solution of (4)-(5) under some assumptions has been presented in Section 4.

\section{Preliminaries}

For the convenience of the readers, we firstly present the necessary definitions from the fractional calculus theory and functional analysis. These definitions and results can be found in the literature $[3,7,38]$.

Let $C[0,1]$ be the Banach space of all continuous real functions defined on $[0,1]$ with the norm

$$
\|u\|=: \max \{|u(t)|: t \in[0,1]\} .
$$

Let $C_{r}[0,1], r \geq 0$, be the space of all functions $f$ such that $t^{r} u(t) \in C[0,1]$ which is a Banach space when endowed with the norm

$$
\|u\|_{r}=: \max \left\{t^{r}|u(t)|: t \in[0,1]\right\} .
$$

Definition 1. For a function $u$ defined on an interval $[a, b]$, the Riemann-Liouville fractional integral of $u$ of order $\alpha>0$ is defined by

$$
D_{a^{+}}^{-\alpha} u(t)=\frac{1}{\Gamma(\alpha)} \int_{a}^{t}(t-s)^{\alpha-1} u(s) d s, \quad t>a,
$$

and Riemann-Liouville fractional derivative of $u(t)$ of order $\alpha>0$ defined by

$$
D_{a^{+}}^{\alpha} u(t)=\frac{d^{n}}{d t^{n}}\left\{D_{a^{+}}^{-(\alpha-n)} u(t)\right\}, \quad n-1<\alpha \leq n,
$$

provided that the right-hand side of the pervious equation is pointwise defined on $(a,+\infty)$.
We denote $D_{a^{+}}^{\alpha} u(t)$ as $D_{a}^{\alpha} y(t)$ and $D_{a^{+}}^{-\alpha} u(t)$ as $D_{a}^{-\alpha} u(t)$. Further $D_{0^{+}}^{\alpha} u(t)$ and $D_{0^{+}}^{-\alpha} u(t)$ are referred as $D^{\alpha} u(t)$ and $D^{-\alpha} u(t)$, respectively.

Definition 2. A two-parameter function of the Mittag-Leffler type is defined by

$$
E_{\alpha, \beta}(z)=\sum_{k=0}^{\infty} \frac{z^{k}}{\Gamma(\alpha k+\beta)}, \quad(\alpha>0, \beta>0) .
$$

Definition 3. The beta function is usually defined by

$$
\begin{aligned}
& B(z, w)=\int_{0}^{1} \tau^{z-1}(1-\tau)^{w-1} d \tau, \\
&(\operatorname{Re}(z)>0, \operatorname{Re}(w)>0) .
\end{aligned}
$$

and we have also the following expression for the beta function:

$$
B(z, w)=\frac{\Gamma(z) \Gamma(w)}{\Gamma(z+w)}
$$

Theorem 4 (Arzela-Ascoli's theorem). A subset of $C[a, b]$ is compact if and only if it is closed, bounded, and equicontinuous.

Theorem 5 (Banach's fixed point theorem). Consider a metric space $X=(X, d)$, where $X \neq \emptyset$. Suppose that $X$ is complete and $T: X \rightarrow X$ is a contraction on $X$. Then $T$ has precisely one fixed point.

Theorem 6 (Krasnoselskii's fixed point theorem). Let $B$ be a nonempty closed convex subset of a Banach space $(X,\|\cdot\|)$. Suppose that $T_{1}$, and $T_{2}$ map $B$ into $X$ such that

(1) for any $x, y \in B$ we have $T_{1} x+T_{2} y \in B$,

(2) $T_{1}$ is a contraction,

(3) $T_{2}$ is continuous and $T_{2}(B)$ is contained in a compact set.

Then there exists $z \in B$ such that $z=T_{1} z+T_{2} z$.

\section{Uniqueness of Solution}

In this section we prove (2) with boundary condition (3) and another condition on $f$ has a unique solution. Before proving, we need to introduce some notations that will be provided in the following.

Let $\Omega=\left\{u:[-\tau, 1] \rightarrow \mathbb{R}, u \in C_{1-\alpha}[0,1]\right\}$. Consider the operator $N: \Omega \rightarrow \Omega$ defined by

$$
N u(t)= \begin{cases}\phi(t), & t \in[-\tau, 0] \\ \int_{0}^{1} G_{1, \alpha}(t, s) f\left(s, u_{s}\right) d s, & t \in(0,1),\end{cases}
$$


where

$$
G_{1, \alpha}(t, s)=\left\{\begin{array}{l}
\frac{\Gamma(\alpha) E_{\alpha, \alpha}\left(t^{\alpha}\right) E_{\alpha, \alpha}\left((1-s)^{\alpha}\right) t^{\alpha-1}(1-s)^{\alpha-1}}{1-\Gamma(\alpha) E_{\alpha, \alpha}(1)} \\
+(t-s)^{\alpha-1} E_{\alpha, \alpha}\left((t-s)^{\alpha}\right) \\
0 \leq s \leq t \leq 1, \\
\frac{\Gamma(\alpha) E_{\alpha, \alpha}\left(t^{\alpha}\right) E_{\alpha, \alpha}\left((1-s)^{\alpha}\right) t^{\alpha-1}(1-s)^{\alpha-1}}{1-\Gamma(\alpha) E_{\alpha, \alpha}(1)} \\
0 \leq t \leq s \leq 1,
\end{array}\right.
$$

is given in Section 4 . Let $x(\cdot):[-\tau, 1] \rightarrow \mathbb{R}$ be the function defined by

$$
x(t)= \begin{cases}0, & t \in(0,1) \\ \phi(t), & t \in[-\tau, 0]\end{cases}
$$

For each $z \in C_{1-\alpha}([0,1], \mathbb{R})$ with $\lim _{t \rightarrow 0^{+}} t^{1-\alpha} z(t)=c$ we denote $\bar{z}$ the function defined by

$$
\bar{z}(t)= \begin{cases}z(t), & t \in(0,1) \\ 0, & t \in[-\tau, 0]\end{cases}
$$

If $u(\cdot)$ satisfies the integral equation,

$$
u(t)=\int_{0}^{1} G_{1, \alpha}(t, s) f\left(s, u_{s}\right) d s
$$

we can decompose $u(\cdot)$ as $u(t)=\bar{z}(t)+x(t), 0<t<1$, which implies $u_{t}=\bar{z}_{t}+x_{t}$ for every $0<t<1$, and function $z(\cdot)$ satisfies

$$
z(t)=\int_{0}^{1} G_{1, \alpha}(t, s) f\left(s, \bar{z}_{s}+x_{s}\right) d s
$$

Set $C_{0}=\left\{z \in\left(C_{1-\alpha}[0,1], \mathbb{R}\right): \lim _{t \rightarrow 0^{+}} t^{1-\alpha} z(t)=c\right\} . C_{0}$ is Banach space with the norm $\|\cdot\|_{r}$. Let $T: C_{0} \rightarrow C_{0}$ be defined by

$$
T z(t)=\int_{0}^{1} G_{1, \alpha}(t, s) f\left(s, \bar{z}_{s}+x_{s}\right) d s, \quad 0<t<1 .
$$

Note that as operator $N$ has a fixed point, equivalently $T$ has a fixed point and so instead we try to prove that $T$ has a fixed point.

Theorem 7. Assume that there exists a constant $K>0$ such that $|f(t, u)-f(t, v)| \leq K|u-v|$ for each $t \in[0,1]$ and all $u, v \in \mathbb{R}$. Then the problem (2)-(3) has a unique solution in $C_{0} \subseteq C_{1-\alpha}[0,1]$ provided that

$$
K\left(\frac{\Gamma(\alpha) E_{\alpha, \alpha}(1)}{\left|1-\Gamma(\alpha) E_{\alpha, \alpha}(1)\right|}+1\right) E_{\alpha, \alpha}(1) \frac{(\Gamma(\alpha))^{2}}{\Gamma(2 \alpha)}<1
$$

Proof. We prove that $T: C_{0} \rightarrow C_{0}$ is a contraction map. For each $t \in[0,1]$ and for $z_{1}, z_{2} \in C_{0}$ we have

$$
\begin{aligned}
& t^{1-\alpha}\left|T z_{1}(t)-T z_{2}(t)\right| \\
& \leq t^{1-\alpha} \int_{0}^{1}\left|G_{1, \alpha}(t, s)\right| \\
& \quad \times\left|f\left(s, \bar{z}_{1 s}+x_{s}\right)-f\left(s, \bar{z}_{2 s}+x_{s}\right)\right| d s \\
& \leq K t^{1-\alpha} \int_{0}^{1}\left|G_{1, \alpha}(t, s)\right|\left|\bar{z}_{1 s}-\bar{z}_{2 s}\right| d s
\end{aligned}
$$

using the definition of $\|\cdot\|_{1-\alpha}$ we get

$$
\begin{aligned}
& \left\|T z_{1}(t)-T z_{2}(t)\right\|_{1-\alpha} \\
& \leq \max _{t \in[0,1]}\left\{t^{1-\alpha} \int_{0}^{1}\left|G_{1, \alpha}(t, s)\right| s^{\alpha-1} d s\right\} \\
& \quad \times\left\|z_{1}-z_{2}\right\|_{1-\alpha} .
\end{aligned}
$$

Moreover,

$$
\begin{aligned}
& t^{1-\alpha} \int_{0}^{1}\left|G_{1, \alpha}(t, s)\right| s^{\alpha-1} d s \\
& \quad \leq\left(\frac{\Gamma(\alpha) E_{\alpha, \alpha}(1)}{\left|1-\Gamma(\alpha) E_{\alpha, \alpha}(1)\right|}+1\right) E_{\alpha, \alpha}(1) \frac{\Gamma(\alpha)^{2}}{\Gamma(2 \alpha)}
\end{aligned}
$$

Indeed we have

$$
\begin{aligned}
t^{1-\alpha} & \int_{0}^{1}\left|G_{1, \alpha}(t, s)\right| s^{\alpha-1} d s \\
\leq & \frac{\Gamma(\alpha)\left|E_{\alpha, \alpha}\left(t^{\alpha}\right)\right|}{\left|1-\Gamma(\alpha) E_{\alpha, \alpha}(1)\right|} \\
& \times \sum_{i=0}^{\infty} \frac{1}{\Gamma(\alpha i+\alpha)} \int_{0}^{1}(1-s)^{\alpha-1} s^{\alpha-1} d s \\
& +t^{\alpha-1} \sum_{i=0}^{\infty} \frac{1}{\Gamma(\alpha i+\alpha)} \\
& \times \int_{0}^{t}(t-s)^{\alpha-1} s^{\alpha-1} d s \frac{\Gamma(\alpha)\left|E_{\alpha, \alpha}\left(t^{\alpha}\right)\right|}{\left|1-\Gamma(\alpha) E_{\alpha, \alpha}(1)\right|} \\
& \times E_{\alpha, \alpha}(1) \int_{0}^{1}(1-s)^{\alpha-1} s^{\alpha-1} d s \\
& +t^{1-\alpha} E_{\alpha, \alpha}(1) \int_{0}^{t}(t-s)^{\alpha-1} s^{\alpha-1} d s .
\end{aligned}
$$

Note that

$$
\int_{0}^{t} s^{\alpha-1}(t-s)^{\alpha-1} d s=t^{2 \alpha-1} \frac{\Gamma(\alpha)^{2}}{\Gamma(2 \alpha)}
$$


Hence we have

$$
\begin{aligned}
t^{1-\alpha} & \int_{0}^{1}\left|G_{1, \alpha}(t, s)\right| s^{\alpha-1} d s \\
\leq & \frac{\Gamma(\alpha)\left|E_{\alpha, \alpha}\left(t^{\alpha}\right)\right|}{\left|1-\Gamma(\alpha) E_{\alpha, \alpha}(1)\right|} E_{\alpha, \alpha}(1) \frac{\Gamma(\alpha)^{2}}{\Gamma(2 \alpha)} \\
& +t^{1-\alpha} E_{\alpha, \alpha}(1) t^{2 \alpha-1} \frac{\Gamma(\alpha)^{2}}{\Gamma(2 \alpha)} \\
= & \left(\frac{\Gamma(\alpha)\left|E_{\alpha, \alpha}\left(t^{\alpha}\right)\right|}{\left|1-\Gamma(\alpha) E_{\alpha, \alpha}(1)\right|}+t^{\alpha}\right) E_{\alpha, \alpha}(1) \frac{(\Gamma(\alpha))^{2}}{\Gamma(2 \alpha)} .
\end{aligned}
$$

Using (22) and (23) we get

$$
\begin{aligned}
\left\|T z_{1}-T z_{2}\right\|_{1-\alpha} \leq & K\left(\frac{\Gamma(\alpha) E_{\alpha, \alpha}(1)}{\left|1-\Gamma(\alpha) E_{\alpha, \alpha}(1)\right|}+1\right) \\
& \times E_{\alpha, \alpha}(1) \frac{\Gamma(\alpha)^{2}}{\Gamma(2 \alpha)}\left\|z_{1}-z_{2}\right\|_{1-\alpha} .
\end{aligned}
$$

This completes the proof.

\section{Existence of Solution}

In this section, by using Krasnoselskii's theorem, we discuss the existence solution of (4) under some assumptions on $f$ and further conditions. Before proving this theorem, we prove the following lemma which will be used in the next theorem.

Lemma 8. Consider the following nonlinear fractional differential equation of the form

$$
D^{\alpha} u(t)=u(t)+h(t), \quad 0<t<1,0<\alpha<1,
$$

with periodic boundary conditions

$$
\begin{gathered}
u(0)=\lim _{t \rightarrow 0^{+}} t^{1-\alpha} u(t)=c=u(1), \\
\left.D^{-(1-\alpha)} u(t)\right|_{t=0}=c \Gamma(\alpha),
\end{gathered}
$$

where $h$ is a continuous function. Then the periodic boundary value problem (28)-(29) is equivalent to an integral equation given by $u(t)=\int_{0}^{1} G_{1, \alpha}(t, s) h(s) d s \in C_{1-\alpha}[0,1]$, where

$$
G_{1, \alpha}(t, s)=\left\{\begin{array}{l}
\frac{\Gamma(\alpha) E_{\alpha, \alpha}\left(t^{\alpha}\right) E_{\alpha, \alpha}\left((1-s)^{\alpha}\right) t^{\alpha-1}(1-s)^{\alpha-1}}{1-\Gamma(\alpha) E_{\alpha, \alpha}(1)} \\
+(t-s)^{\alpha-1} E_{\alpha, \alpha}\left((t-s)^{\alpha}\right) \\
0 \leq s \leq t \leq 1, \\
\frac{\Gamma(\alpha) E_{\alpha, \alpha}\left(t^{\alpha}\right) E_{\alpha, \alpha}\left((1-s)^{\alpha}\right) t^{\alpha-1}(1-s)^{\alpha-1}}{1-\Gamma(\alpha) E_{\alpha, \alpha}(1)} \\
0 \leq t \leq s \leq 1 .
\end{array}\right.
$$

Proof. We consider the following fractional differential equation:

$$
D^{\alpha} y(t)-y(t)=h(t), \quad(0<t<1,0<\alpha<1),
$$

with

$$
\left[D^{\alpha-1} y(t)\right]_{t=0}=b_{1}=c \Gamma(\alpha)
$$

where $\lim _{t \rightarrow 0^{+}} t^{1-\alpha} y(t)=c$. Laplace transform of (31) yields

$$
s^{\alpha} Y(s)-Y(s)=H(s)+b_{1}
$$

from which

$$
Y(s)=\frac{H(s)}{s^{\alpha}-1}+\frac{b_{1}}{s^{\alpha}-1}
$$

and the inverse Laplace transform gives the solution

$$
\begin{aligned}
y(t)= & b_{1} t^{\alpha-1} E_{\alpha, \alpha}\left(t^{\alpha}\right) \\
& +\int_{0}^{t}(t-s)^{\alpha-1} E_{\alpha, \alpha}(t-s)^{\alpha} h(s) d s .
\end{aligned}
$$

Hence we have

$$
\begin{aligned}
y(t)= & c \Gamma(\alpha) t^{\alpha-1} E_{\alpha, \alpha}\left(t^{\alpha}\right) \\
& +\int_{0}^{t}(t-s)^{\alpha-1} E_{\alpha, \alpha}(t-s)^{\alpha} h(s) d s .
\end{aligned}
$$

Therefore,

$$
\begin{aligned}
y(1)= & c \Gamma(\alpha)+E_{\alpha, \alpha}(1) \\
& +\int_{0}^{1}(1-s)^{\alpha-1} E_{\alpha, \alpha}\left((1-s)^{\alpha}\right) h(s) d s,
\end{aligned}
$$

which leads to

$$
\begin{aligned}
c(1- & \left.\Gamma(\alpha) E_{\alpha, \alpha}(1)\right) \\
= & \int_{0}^{1}(1-s)^{\alpha-1} E_{\alpha, \alpha}\left((1-s)^{\alpha}\right) h(s) d s ;
\end{aligned}
$$

since $\Gamma(\alpha) E_{\alpha, \alpha}(1) \neq 1$ we have

$$
c=\frac{1}{1-\Gamma(\alpha) E_{\alpha, \alpha}(1)} \int_{0}^{1}(1-s)^{\alpha-1} E_{\alpha, \alpha}\left((1-s)^{\alpha}\right) h(s) d s
$$

Then the solution of the problem (28)-(29) is given by

$$
\begin{aligned}
y(t)= & \frac{\Gamma(\alpha)}{1-\Gamma(\alpha) E_{\alpha, \alpha}(1)} t^{\alpha-1} E_{\alpha, \alpha}\left(t^{\alpha}\right) \\
& \times \int_{0}^{1}(1-s)^{\alpha-1} E_{\alpha, \alpha}\left((1-s)^{\alpha}\right) h(s) d s \\
& +\int_{0}^{t}(t-s)^{\alpha-1} E_{\alpha, \alpha}\left((t-s)^{\alpha}\right) h(s) d s .
\end{aligned}
$$

This completes the proof. 
Now we prove our main result using Lemma 8 and two more assumptions which follow next.

(H1) We assume that $f(t, u, v)$ can be written as $f_{1}(t, u)+$ $f_{2}(t, u, v)$, where $f_{1}, f_{2}$ are Lipschitz continuous. Moreover assume that the function $f_{1}, f_{2}$ satisfies the following relations:

$$
\begin{gathered}
\left|f_{1}(t, u(t))\right| \leq M_{1}\|u(t)\|_{1-\alpha}, \\
\left|f_{2}(t, u(t), v(t))\right| \leq M_{2}\|u(t)\|_{1-\alpha}\|v(t)\|_{1-\alpha} .
\end{gathered}
$$

(H2) Let $\Omega=C_{1-\alpha}([-\tau, 1], \mathbb{R})$ denote collection of the space of all function $u$ such that $t^{1-\alpha} u(t) \in C[0,1]$. Define the set $B=\left\{u \in \Omega: t^{1-\alpha}|u| \leq r\right\}$, where $r$ satisfies

$$
\begin{aligned}
& \left(M_{1}\|u\|_{1-\alpha}+M_{2}\|u\|_{1-\alpha}^{2}\right) \\
& \quad \times\left(\frac{|\Gamma(\alpha)| E_{\alpha, \alpha}\left(t^{\alpha}\right) \mid}{\left|1-\Gamma(\alpha) E_{\alpha, \alpha}(1)\right|} E_{\alpha, \alpha+1}(1)+t E_{\alpha, \alpha+1}\left(t^{\alpha}\right)\right) \leq r .
\end{aligned}
$$

Furthermore we assume that

$$
L_{f_{1}}\left(\frac{\Gamma(\alpha) E_{\alpha, \alpha}(1)}{\left|1-\Gamma(\alpha) E_{\alpha, \alpha}(1)\right|}+1\right) E_{\alpha, \alpha}(1) \frac{(\Gamma(\alpha))^{2}}{\Gamma(2 \alpha)}<1,
$$

where $L_{f_{1}}$ is a Lipschitz constant of $f$.

Theorem 9. If the assumptions (H1) and (H2) satisfied, then the problem (4) with periodic boundary value condition (5) has at least one solution.

Proof. (i) Note that by Lemma 8, (4)-(5) is equivalent to integral equation (17). Define $T_{1}, T_{2}: \Omega \rightarrow \Omega$ by

$$
\begin{gathered}
T_{1} u(t)=\int_{0}^{1} G_{1, \alpha}(t, s) f_{1}(s, u(s)) d s \\
T_{2} u(t)=\int_{0}^{1} G_{1, \alpha}(t, s) f_{2}(s, u(s), u(s-\tau)) d s .
\end{gathered}
$$

For $u \in B$ we have,

$$
\begin{aligned}
& t^{1-\alpha}\left|T_{1} u(t)+T_{2} u(t)\right| \\
& \leq t^{1-\alpha} \int_{0}^{1}\left|G_{1, \alpha}(t, s)\right| \\
& \quad \times \mid f_{1}(s, u(s)) \\
& \quad+f_{2}(s, u(s), u(s-\tau)) \mid d s \\
& \leq\left(M_{1}\|u\|_{1-\alpha}+M_{2}\|u\|_{1-\alpha}^{2}\right) t^{1-\alpha} \\
& \quad \times \int_{0}^{1}\left|G_{1, \alpha}(t, s)\right| d s
\end{aligned}
$$

$$
\begin{aligned}
& \leq\left(M_{1}\|u\|_{1-\alpha}+M_{2}\|u\|_{1-\alpha}^{2}\right) \\
& \times\left(\frac{\Gamma(\alpha)\left|E_{\alpha, \alpha}\left(t^{\alpha}\right)\right|}{\left|1-\Gamma(\alpha) E_{\alpha, \alpha}(1)\right|} E_{\alpha, \alpha+1}(1)\right. \\
&\left.+t E_{\alpha, \alpha+1}\left(t^{\alpha}\right)\right) \leq r .
\end{aligned}
$$

(ii) We will prove that $T_{1}$ is a contraction:

$$
\begin{aligned}
& \mid T_{1} u-T_{1} v \|_{1-\alpha} \\
& =t^{1-\alpha}\left|T_{1} u(t)-T_{1} v(t)\right|, \\
& \leq t^{1-\alpha} \int_{0}^{1}\left|G_{1, \alpha}(t, s)\right| \\
& \quad \times\left|f_{1}(s, u(s))-f_{1}(s, v(s))\right| d s, \\
& \leq L_{f_{1}} t^{1-\alpha} \int_{0}^{1}\left|G_{1, \alpha}(t, s)\right| u-v \mid d s, \\
& \leq L_{f_{1}} t^{1-\alpha} \int_{0}^{1}\left|G_{1, \alpha}(t, s) s^{\alpha-1} s^{1-\alpha}\right| u-v \mid d s \\
& \leq L_{f_{1}}\left(\frac{\Gamma(\alpha) E_{\alpha, \alpha}(1)}{\left|1-\Gamma(\alpha) E_{\alpha, \alpha}(1)\right|}+1\right) \\
& \quad \times E_{\alpha, \alpha}(1) \frac{(\Gamma(\alpha))^{2}}{\Gamma(2 \alpha)}\|u-v\|_{1-\alpha} .
\end{aligned}
$$

Then $T_{1}$ is a contraction.

(iii) Finally we prove that $T_{2}$ is continuous and $T_{2}(B)$ is contained in a compact set. To prove the continuity of $T_{2}$ let us consider a sequence $u_{n}$ converging to $u$. Taking the norm of $T_{2} u_{n}(t)-T_{2} u(t)$ we have

$$
\begin{aligned}
& \left\|T_{2} u_{n}(t)-T_{2} u(t)\right\|_{1-\alpha} \\
& =t^{1-\alpha}\left|T_{2} u_{n}(t)-T_{2} u(t)\right| \\
& \leq t^{1-\alpha} \int_{0}^{1}\left|G_{1, \alpha}(t, s)\right| \\
& \times \mid f_{2}\left(s, u_{n}(s), u_{n}(s-\tau)\right) \\
& -f_{2}(s, u(s), u(s-\tau)) \mid d s \\
& \leq t^{1-\alpha} L_{f_{2}} \int_{0}^{1}\left|G_{1, \alpha}(t, s)\right| \\
& \times\left(\left|u_{n}(s)-u(s)\right|\right. \\
& \left.+u_{n}(s-\tau)-u(s-\tau) \mid\right) d s \\
& \leq 2 L_{f_{2}} t^{1-\alpha} \int_{0}^{1}\left|G_{1, \alpha}(t, s)\right| s^{\alpha-1} \\
& \times d s\left\|u_{n}-u\right\|_{1-\alpha} .
\end{aligned}
$$

Hence whenever $u_{n} \rightarrow u$ we have $T_{2} u_{n} \rightarrow T_{2} u$. This proves the continuity of $T_{2}$. 
On the other hand for $0 \leq t_{1}<t_{2} \leq 1$ and $u \in B$ we have

$$
\begin{aligned}
& \left|t_{1}^{1-\alpha} T_{2} u\left(t_{1}\right)-t_{2}^{1-\alpha} T_{2} u\left(t_{2}\right)\right| \\
& =\mid t_{1}^{1-\alpha} \int_{0}^{1} G_{1, \alpha}\left(t_{1}, s\right) f_{2}(s, u(s), u(s-\tau)) d s \\
& -t_{2}^{1-\alpha} \int_{0}^{1} G_{1, \alpha}\left(t_{2}, s\right) f_{2}(s, u(s), u(s-\tau)) d s \\
& \leq \mid \frac{\Gamma(\alpha) E_{\alpha, \alpha}\left(t_{1}^{\alpha}\right)}{1-\Gamma(\alpha) E_{\alpha, \alpha}(1)} \\
& \times \int_{0}^{t_{1}} E_{\alpha, \alpha}\left((1-s)^{\alpha}\right)(1-s)^{\alpha-1} \\
& \times f_{2}(s, u(s), u(s-\tau)) d s \\
& +t_{1}^{1-\alpha} \int_{0}^{t_{1}} E_{\alpha, \alpha}\left(\left(t_{1}-s\right)^{\alpha}\right)\left(t_{1}-s\right)^{\alpha-1} \\
& \times f_{2}(s, u(s), u(s-\tau)) d s \\
& -\frac{\Gamma(\alpha) E_{\alpha, \alpha}\left(t_{2}^{\alpha}\right)}{1-\Gamma(\alpha) E_{\alpha, \alpha}(1)} \\
& \times \int_{0}^{t_{1}} E_{\alpha, \alpha}\left((1-s)^{\alpha}\right)(1-s)^{\alpha-1} \\
& \times f_{2}(s, u(s), u(s-\tau)) \\
& -t_{2}^{1-\alpha} \int_{0}^{t_{1}} E_{\alpha, \alpha}\left(\left(t_{2}-s\right)^{\alpha}\right)\left(t_{2}-s\right)^{\alpha-1} \\
& \times f_{2}(s, u(s), u(s-\tau)) \\
& +\frac{\Gamma(\alpha) E_{\alpha, \alpha}\left(t_{1}^{\alpha}\right)}{1-\Gamma(\alpha) E_{\alpha, \alpha}(1)} \\
& \times \int_{t_{1}}^{t_{2}} E_{\alpha, \alpha}\left((1-s)^{\alpha}\right)(1-s)^{\alpha-1} \\
& \times f(s, u(s), u(s-\tau)) d s \\
& -\frac{\Gamma(\alpha) E_{\alpha, \alpha}\left(t_{2}^{\alpha}\right)}{1-\Gamma(\alpha) E_{\alpha, \alpha}(1)} \\
& \times \int_{t_{1}}^{t_{2}} E_{\alpha, \alpha}\left((1-s)^{\alpha}\right)(1-s)^{\alpha-1} \\
& \times f_{2}(s, u(s), u(s-\tau)) d s \\
& -t_{2}^{1-\alpha} \int_{t_{1}}^{t_{2}} E_{\alpha, \alpha}\left(t_{2}-s\right)^{\alpha}\left(t_{2}-s\right)^{\alpha-1} \\
& \times f_{2}(s, u(s), u(s-\tau)) d s \\
& +\frac{\Gamma(\alpha) E_{\alpha, \alpha}\left(t_{1}^{\alpha}\right)}{1-\Gamma(\alpha) E_{\alpha, \alpha}(1)}
\end{aligned}
$$

$$
\begin{aligned}
& \times \int_{t_{2}}^{1} E_{\alpha, \alpha}\left((1-s)^{\alpha}\right)(1-s)^{\alpha-1} \\
& \times f_{2}(s, u(s), u(s-\tau)) d s \\
& -\frac{\Gamma(\alpha) E_{\alpha, \alpha}\left(t_{2}^{\alpha}\right)}{1-\Gamma(\alpha) E_{\alpha, \alpha}(1)} \\
& \times \int_{t_{2}}^{1} E_{\alpha, \alpha}\left((1-s)^{\alpha-1}\right)(1-s)^{\alpha-1} \\
& \quad \times f_{2}(s, u(s), u(s-\tau)) d s .
\end{aligned}
$$

Then we have

$$
\begin{aligned}
& \left|t_{1}^{1-\alpha} T_{2} u\left(t_{1}\right)-t_{2}^{1-\alpha} T_{2} u\left(t_{2}\right)\right| \\
& \leq M_{2}\|u\|_{1-\alpha}^{2} \\
& \times\left(\frac{\Gamma(\alpha)}{1-\Gamma(\alpha) E_{\alpha, \alpha}(1)}\left|E_{\alpha, \alpha}\left(t_{1}^{\alpha}\right)-E_{\alpha, \alpha}\left(t_{2}^{\alpha}\right)\right|\right. \\
& \times \int_{0}^{t_{1}}\left|E_{\alpha, \alpha}\left((1-s)^{\alpha}\right)\right|(1-s)^{\alpha-1} d s \\
& +\int_{0}^{t_{1}} \mid t_{1}^{1-\alpha} E_{\alpha, \alpha}\left(\left(t_{1}-s\right)^{\alpha}\right)\left(t_{1}-s\right)^{\alpha-1} \\
& -t_{2}^{1-\alpha}\left(t_{2}-s\right)^{\alpha-1} E_{\alpha, \alpha}\left(\left(t_{2}-s\right)^{\alpha}\right) \mid d s \\
& +\frac{\Gamma(\alpha)}{\left|1-\Gamma(\alpha) E_{\alpha, \alpha}(1)\right|}\left|E_{\alpha, \alpha}\left(t_{1}^{\alpha}\right)-E_{\alpha, \alpha}\left(t_{2}^{\alpha}\right)\right| \\
& \times \int_{t_{1}}^{t_{2}}\left|E_{\alpha, \alpha}\left((1-s)^{\alpha}\right)\right|(1-s)^{\alpha-1} d s \\
& -t_{2}^{1-\alpha} \int_{t_{1}}^{t_{2}}\left|E_{\alpha, \alpha}\left(\left(t_{2}-s\right)^{\alpha}\right)\right|\left(t_{2}-s\right)^{\alpha-1} d s \\
& +\frac{\Gamma(\alpha)}{\left|1-\Gamma(\alpha) E_{\alpha, \alpha}(1)\right|} \\
& \times\left|E_{\alpha, \alpha}\left(t_{1}^{\alpha}\right)-\right| E_{\alpha, \alpha}\left(t_{2}^{\alpha}\right) \mid \\
& \left.\times \int_{t_{2}}^{1}\left|E_{\alpha, \alpha}\left((1-s)^{\alpha}\right)\right|(1-s)^{\alpha-1} d s\right) .
\end{aligned}
$$

Hence we deduce that if $\left|t_{1}-t_{2}\right| \rightarrow 0$ then $\mid t_{1}^{1-\alpha} T_{2}\left(t_{2}\right)-$ $t_{2}^{1-\alpha} T_{2}\left(t_{2}\right) \mid \rightarrow 0$.

Then $\left\{t^{1-\alpha} T_{2} u: u \in B\right\}$ is equicontinuous. Moreover we show that $\left\{t^{1-\alpha} T_{2} u: u \in B\right\}$ is a bounded set in $C[0,1]$. Indeed we have

$$
\begin{aligned}
t^{1-\alpha} & \left|T_{2} u(t)\right| \\
\leq & M_{2}\|u\|_{1-\alpha}^{2} t^{1-\alpha} \int_{0}^{1}\left|G_{1, \alpha}(t, s)\right| d s \\
\leq & M_{2}\|u\|_{1-\alpha}^{2} \\
& \times\left(\frac{\Gamma(\alpha)\left|E_{\alpha, \alpha}\left(t^{\alpha}\right)\right|}{\left|1-\Gamma(\alpha) E_{\alpha, \alpha}(1)\right|} E_{\alpha, \alpha+1}(1)+t E_{\alpha, \alpha+1}\left(t^{\alpha}\right)\right) .
\end{aligned}
$$


Then by Arzela-Ascoli's theorem we conclude that $T_{2}$ is compact. By using Krasnoselskii's theorem there exists $z \in B$ such that $z$ is a fixed point of $T$. This completes the proof.

\section{Conclusions}

We considered two types of nonlinear delay fractional differential equations (FDE) with periodic boundary conditions involving Remann-Liouville fractional derivative possessing with a lower terminal at 0 . In order to obtain the results in this paper, we have shown the existence and the uniqueness of solution for a class of nonlinear delayed FDE by Banach contraction principle. Then using Krasnoselskii's fixed point theorem we established an existence theorem for a different type of the equation that we have proven its uniqueness theorem.

\section{Acknowledgment}

The authors would like thank to the anonymous referees for their valuable comments and suggestions.

\section{References}

[1] W. Boyce and R. Diprima, Elementary Differential Equations and Boundary Value Problems, John Wiley \& Sons, New York, NY, USA, 1997.

[2] F. Moon and M. Johnson, Dynamics and Chaos in Manufacturing Processes, chapter 1, John Wiley \& Sons, New York, NY, USA, 1998.

[3] A. A. Kilbas, H. M. Srivastava, and J. J. Trujillo, Theory and Application of Fractional Differential Equations, Elsevier Science B.V., Amsterdam, The Netherlands, 2006.

[4] S. Das, Functional Fractional Calculus for System Identification and Controls, Springer, Berlin, Germany, 2008.

[5] R. Hilfer, Ed., Applications of Fractional Calculus in Physics, World Scientific, River Edge, NJ, USA, 2000.

[6] N. Heymans and I. Podlubny, "Physical interpretation of initial conditions for fractional differential equations with RiemannLiouville fractional derivatives," Rheologica Acta, vol. 45, no. 5, pp. 765-771, 2006.

[7] I. Podlubny, Fractional Differential Equations, Academic Press, London, UK, 1999.

[8] I. Podlubny, "Geometric and physical interpretation of fractional integration and fractional differentiation," Fractional Calculus \& Applied Analysis, vol. 5, no. 4, pp. 367-386, 2002, Dedicated to the 60th anniversary of Prof. Francesco Mainardi.

[9] I. Podlubny, I. Petráš, B. M. Vinagre, P. O’Leary, and L. Dorčák, "Analogue realizations of fractional-order controllers. Fractional order calculus and its applications," Nonlinear Dynamics, vol. 29, no. 1-4, pp. 281-296, 2002.

[10] J. Sabatier, O. P. Agrawal, and J. A. T. Machado, Advances in Fractional Calculus: Theoretical Developments and Applications in Physics and Engineering, Springer, New York, NY, USA, 2007.

[11] J. T. Machado, V. Kiryakova, and F. Mainardi, "Recent history of fractional calculus," Communications in Nonlinear Science and Numerical Simulation, vol. 16, no. 3, pp. 1140-1153, 2011.

[12] J. T. Machado, A. M. Galhano, and J. J. Trujillo, "On development of fractional calculus during the last fifty years," in Scientometrics, Springer, New York, NY, USA, 2013.
[13] A. El-Sayed, "Fractional order differential equation," Kyungpook Mathematical Journal, vol. 28, no. 2, pp. 18-22, 1988.

[14] A. El-Sayed, "On the fractional differential equations," Applied Mathematics and Computation, vol. 49, no. 2-3, pp. 205-213, 1992.

[15] A. El-Sayed, "Nonlinear functional-differential equations of arbitrary orders," Nonlinear Analysis. Theory, Methods \& Applications. An International Multidisciplinary Journal. Series A: Theory and Methods, vol. 33, no. 2, pp. 181-186, 1998.

[16] R. P. Agarwal, Y. Zhou, and Y. He, "Existence of fractional neutral functional differential equations," Computers \& Mathematics with Applications, vol. 59, no. 3, pp. 1095-1100, 2010.

[17] V. Daftardar-Gejji and A. Babakhani, "Analysis of a system of fractional differential equations," Journal of Mathematical Analysis and Applications, vol. 293, no. 2, pp. 511-522, 2004.

[18] Y. Liu, "Existence of solutions for impulsive differential models on half lines involving Caputo fractional derivatives," Communications in Nonlinear Science and Numerical Simulation, vol. 18, no. 10, pp. 2604-2625, 2013.

[19] D. Delbosco and L. Rodino, "Existence and uniqueness for a nonlinear fractional differential equation," Journal of Mathematical Analysis and Applications, vol. 204, no. 2, pp. 609-625, 1996.

[20] J. Deng and L. Ma, "Existence and uniqueness of solutions of initial value problems for nonlinear fractional differential equations," Applied Mathematics Letters, vol. 23, no. 6, pp. 676$680,2010$.

[21] K. Diethelm and N. J. Ford, "Analysis of fractional differential equations," Journal of Mathematical Analysis and Applications, vol. 265, no. 2, pp. 229-248, 2002.

[22] A. El-Sayed, A. El-Mesiry, and H. El-Saka, "On the fractionalorder logistic equation," Applied Mathematics Letters, vol. 20, no. 7, pp. 817-823, 2007.

[23] K. M. Furati and N.-E. Tatar, "Long time behavior for a nonlinear fractional model," Journal of Mathematical Analysis and Applications, vol. 332, no. 1, pp. 441-454, 2007.

[24] S. B. Hadid, "Local and global existence theorems on differential equations of non-integer order," Journal of Fractional Calculus, vol. 7, pp. 101-105, 1995.

[25] S. Hadid, B. Masaedeh, and S. Momani, "On the existence of maximal and minimal solutions of differential equations of non-integer order," Journal of Fractional Calculus, vol. 9, pp. 4144, 1996.

[26] R. W. Ibrahim and S. Momani, "On the existence and uniqueness of solutions of a class of fractional differential equations," Journal of Mathematical Analysis and Applications, vol. 334, no. 1, pp. 1-10, 2007.

[27] V. Lakshmikantham and A. S. Vatsala, "Basic theory of fractional differential equations," Nonlinear Analysis. Theory, Methods \& Applications. An International Multidisciplinary Journal. Series A: Theory and Methods, vol. 69, no. 8, pp. 2677-2682, 2008.

[28] M. Benchohra, J. Henderson, S. K. Ntouyas, and A. Ouahab, "Existence results for fractional order functional differential equations with infinite delay," Journal of Mathematical Analysis and Applications, vol. 338, no. 2, pp. 1340-1350, 2008.

[29] M. Belmekki, J. J. Nieto, and R. Rodríguez-López, “Existence of periodic solution for a nonlinear fractional differential equation," Boundary Value Problems, vol. 2009, Article ID 324561, 18 pages, 2009. 
[30] S. Abbas, "Existence of solutions to fractional order ordinary and delay differential equations and applications," Electronic Journal of Differential Equations, vol. 2011, no. 09, pp. 1-11, 2011.

[31] V. Lakshmikantham and A. S. Vatsala, "Theory of fractional differential inequalities and applications," Communications in Applied Analysis, vol. 11, no. 3-4, pp. 395-402, 2007.

[32] C. Yu and G. Gao, "Existence of fractional differential equations," Journal of Mathematical Analysis and Applications, vol. 310, no. 1, pp. 26-29, 2005.

[33] S. Abbas, "Pseudo almost automorphic solutions of fractional order neutral differential equation," Semigroup Forum, vol. 81, no. 3, pp. 393-404, 2010.

[34] R. P. Agarwal, M. Benchohra, and S. Hamani, "Boundary value problems for fractional differential equations," Georgian Mathematical Journal, vol. 16, no. 3, pp. 401-411, 2009.

[35] J. Banas and T. Zajcac, "A new approach to the theory of functional integral equations of fractional order," Journal of Mathematical Analysis and Applications, vol. 375, no. 2, pp. 375387, 2011.

[36] C. Giannantoni, "The problem of the initial conditions and their physical meaning in linear differential equations of fractional order," Applied Mathematics and Computation, vol. 141, no. 1, pp. 87-102, 2003.

[37] N. Heymans and I. Podlubny, "Physical interpretation of initialconditions for fractional differential equations with RiemannLiouville fractional derivatives," Rheologica Acta, vol. 45, no. 5, pp. 765-771, 2006.

[38] E. Kreyszig, Introductory Functional Analysis with Applications, John Wiley \& Sons, New York, NY, USA, 1978. 


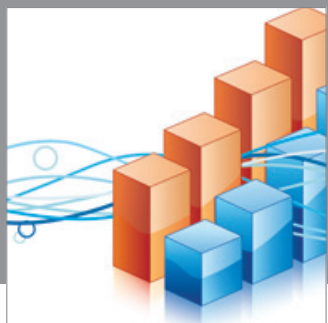

Advances in

Operations Research

mansans

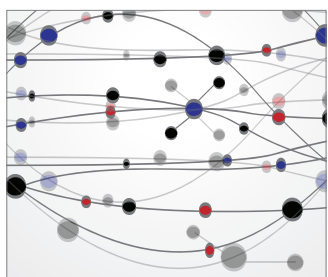

The Scientific World Journal
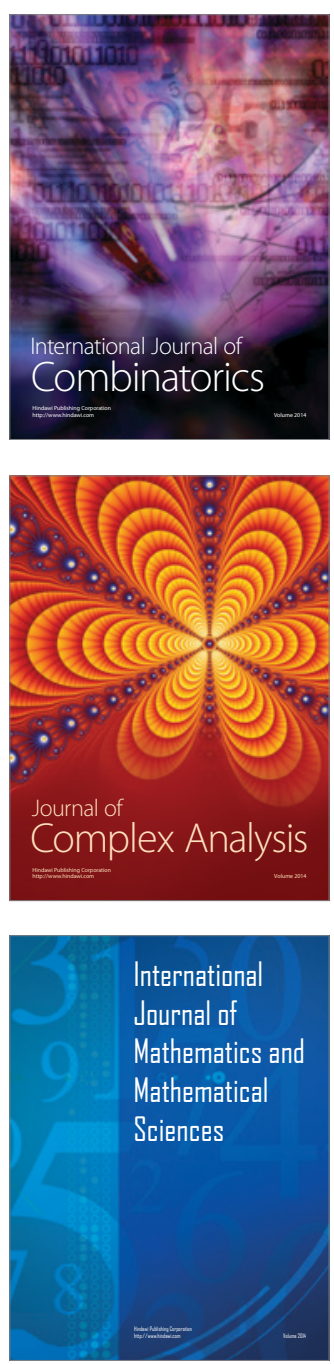
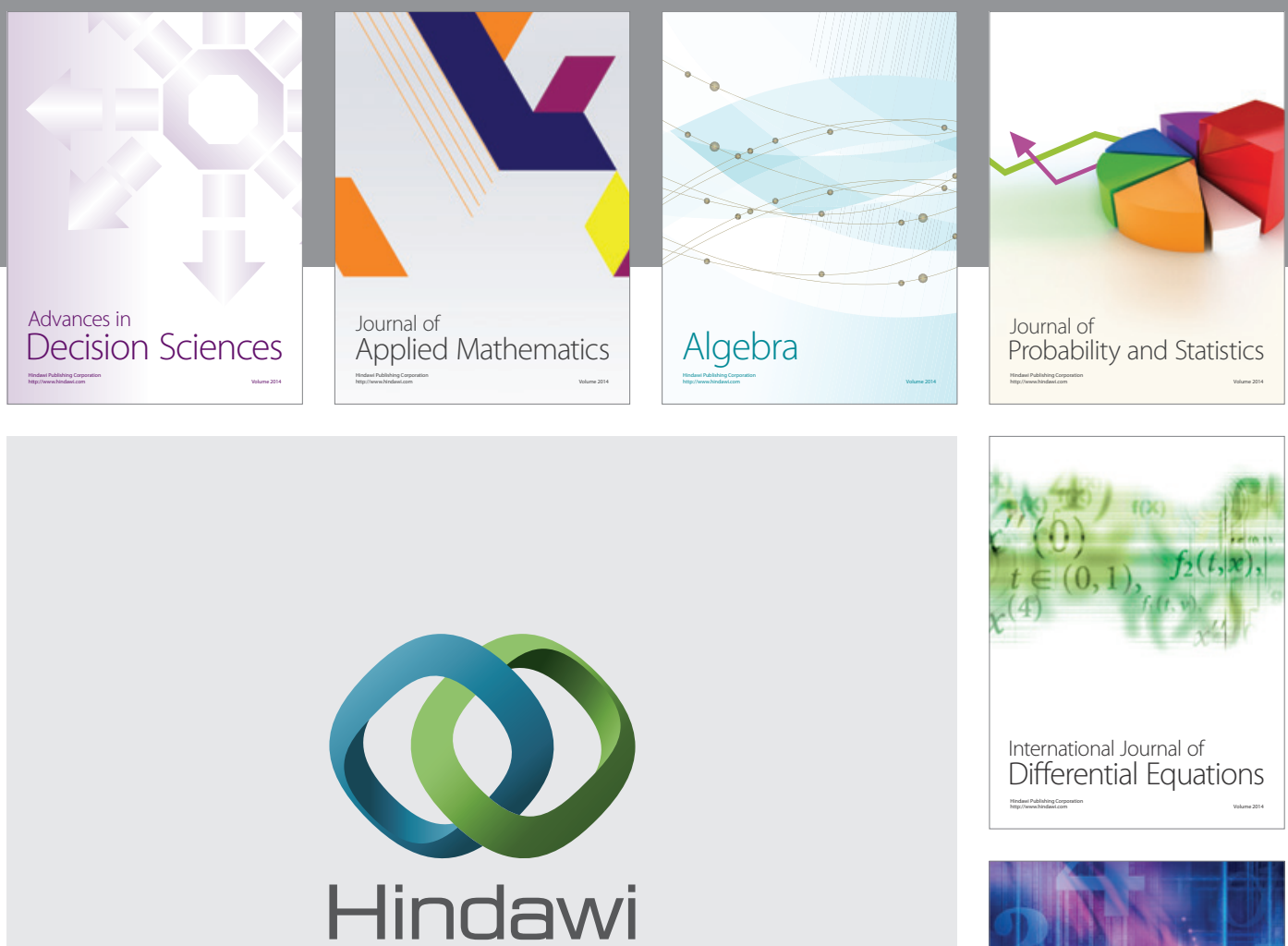

Submit your manuscripts at http://www.hindawi.com
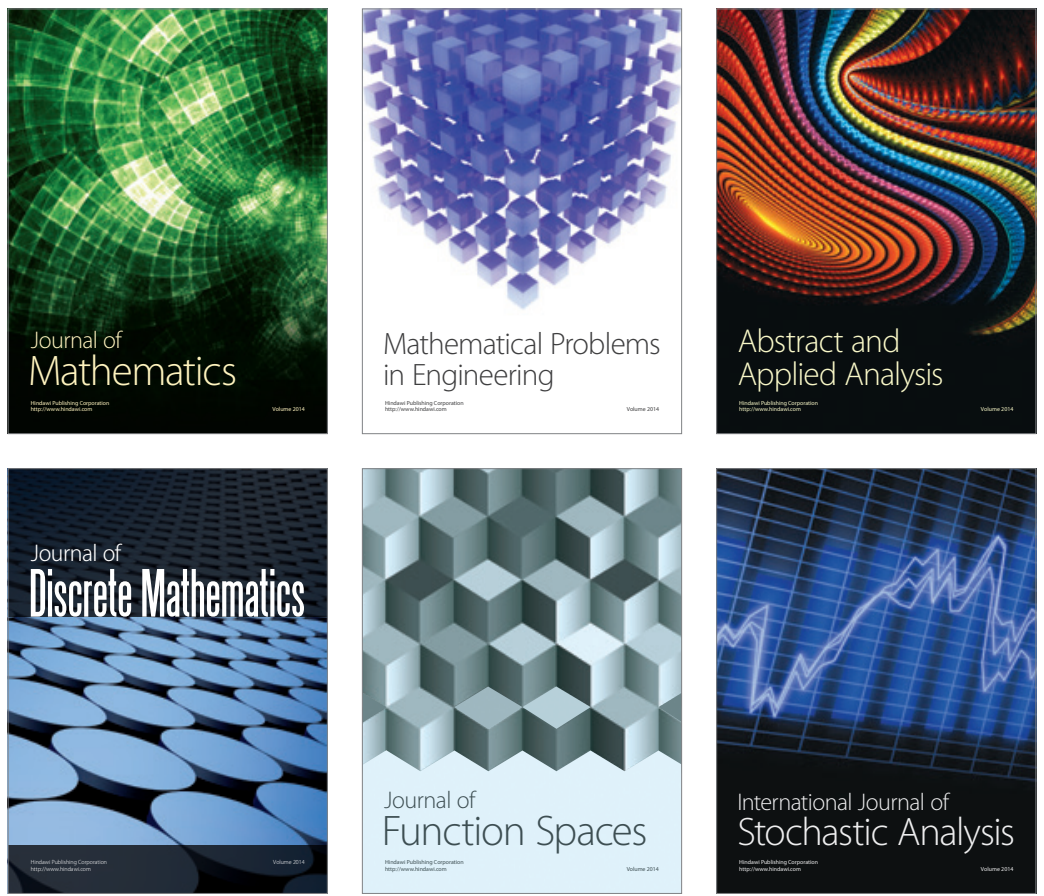

Journal of

Function Spaces

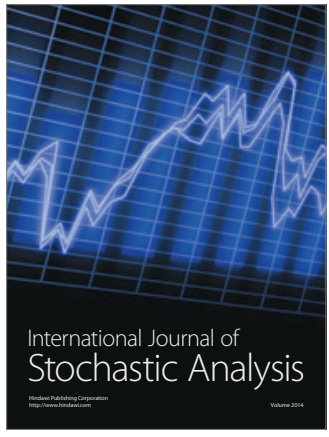

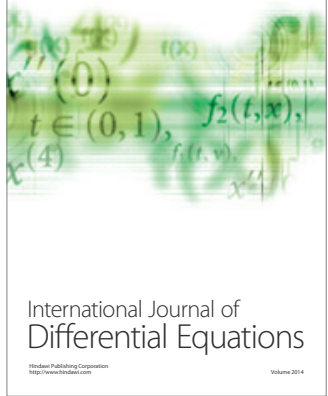
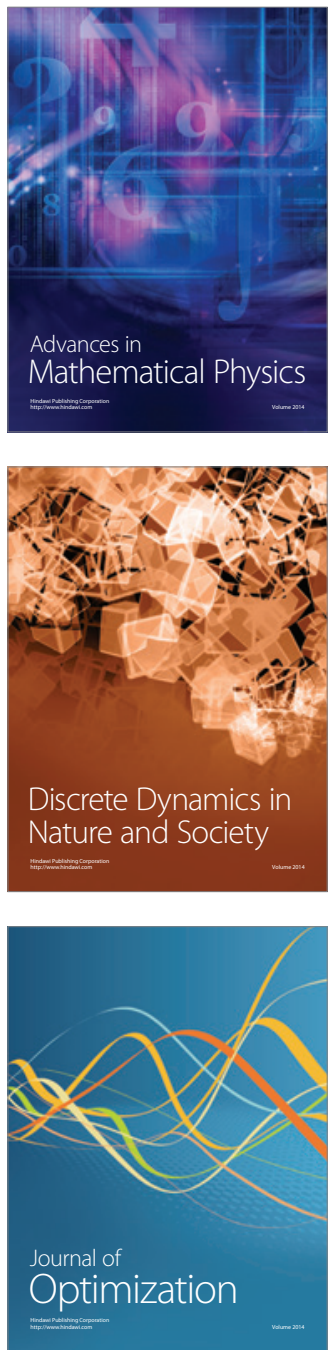